\title{
Ultra thin films of nanocrystalline Ge studied by AFM and interference enhanced Raman scattering ${ }^{\text {II }}$
}

\author{
S BALAJI ${ }^{1}, \mathrm{~S} \mathrm{MOHAN}^{1}$, D V S MUTHU ${ }^{2}$ and A $\mathrm{K} \mathrm{SOOD}^{2, *}$ \\ ${ }^{1}$ Department of Instrumentation, and ${ }^{2}$ Department of Physics, \\ Indian Institute of Science, Bangalore 560 012, India \\ e-mail: asood@physics.iisc.ernet.in
}

\begin{abstract}
Initial growth stages of the ultra thin films of germanium $(\mathrm{Ge})$ prepared by ion beam sputter deposition have been studied using atomic force microscope (AFM) and interference enhanced Raman scattering. The growth of the films follows Volmer-Weber growth mechanism. Analysis of the AFM images shows that Ostwald ripening of the grains occurs as the thickness of the film increases. Raman spectra of the Ge films reveal phonon confinement along the growth direction and show that the misfit strain is relieved for film thickness greater than $4 \mathrm{~nm}$.
\end{abstract}

Keywords. Ion beam sputtering; ultra thin Ge films; interference enhanced Raman spectroscopy; phonon confinement; atomic force microscopy.

\section{Introduction}

There is a considerable interest in recent years to understand growth of germanium $(\mathrm{Ge})$ films on silicon $(\mathrm{Si})$ because of their usefulness in optoelectronic devices based on strained layer superlattices, ${ }^{1}$ and strain-induced self-assembled quantum dots. ${ }^{1}$ The latter have also been observed when Ge is grown on polymer surfaces, opening up the possibility of developing quantum lasers, single electron transistors and various other applications. ${ }^{2}$ Stranski-Krastanov (SK) growth mode is the main mechanism which is observed in the growth of the Ge on Si. In this growth mode, a wetting layer is first formed up to a certain critical thickness $\left(t_{\text {cri }}\right)$, which is strained because of the $\sim 4 \%$ lattice mismatch between $\mathrm{Ge}$ and $\mathrm{Si}$. After $t_{\text {cri }}$, the strain in the uniform two-dimensional layers is relaxed by the formation of three dimensional islands. ${ }^{3}$ Depending on the surface orientation of $\mathrm{Si}$ and substrate temperature $\left(T_{S}\right)$, the critical thickness and the shape of the islands are found to vary. The critical thickness is $\sim 3-7$ monolayers (ML) for Ge on $\mathrm{Si}(001)^{4}$ whereas it increases up to $10 \mathrm{ML}$ for $(015)$ surface. ${ }^{5}$ The $3 \mathrm{D}$ islands can be of different shapes like triangular on $\mathrm{Si}(111)^{6}$, rectangular on $\operatorname{Si}(001)\left(\text { at } T_{s}=700^{\circ} \mathrm{C}\right)^{4}$ and hut clusters on $\mathrm{Si}(015) .{ }^{5}$ Bimodal distribution of the islands were also observed for the Ge film prepared on $\mathrm{Si}(001)$ at a $T_{s}$ of $600^{\circ} \mathrm{C}$. $^{1}$ The strain in the initial layers of Ge have been measured by AFM force measurements on $\mathrm{Si}(111)^{6}$ and on $\mathrm{Si}(100){ }^{4}$ Waltz et $a l^{6}$ have observed compressive stress $\sim 6 \mathrm{GPa}$ for Ge film of $0.8 \mathrm{~nm}$ thickness. TEM studies have also been performed to study the initial growth of Ge films on $\mathrm{Si}^{7}$

In the initial growth of thin films, three types of growth can occur, depending on the surface free energy of the substrate $\left(\sigma_{s}\right)$, surface free energy of the epilayer $\left(\sigma_{f}\right)$ and the interface free energy $\left(\gamma_{i}\right)$. If $\left(\sigma_{f}+\gamma_{i}\right)>\sigma_{s}$, the three dimensional island growth, called

\footnotetext{
${ }^{\mathbb{I}}$ Dedicated to Professor C N R Rao on his 70th birthday

*For correspondence
} 
Volmer-Weber (VW) growth is favoured. If $\left(\sigma_{f}+\gamma_{1}\right)<\sigma_{s}$, the layer by layer growth, called Frank van der Merwe growth can occur. If the film is strained due to lattice mismatch, the SK growth mode is preferred. ${ }^{8}$ The growth of Ge on polymer substrate occurs via VW growth mode because of the non-wetting interface. ${ }^{2}$ A similar growth mode is favoured for the formation of semiconductor films on insulator (SOI) because the surface free energy on insulators are considerably lower when compared with semiconductor substrates. SOI structures are gaining importance from the point of view of nano devices and single electron transistors. ${ }^{9}$ In this context, initial growth stages of $\mathrm{Ge}$ ultra thin films on insulating ceria $\left(\mathrm{CeO}_{2}\right)$ surface are of considerable interest as the lattice constants of ceria are same as that of Si, giving a lattice mismatch of $\sim 4 \%$.

In this paper, single ion beam sputtered Ge films on ceria have been studied using AFM and interference enhanced Raman spectroscopy (IERS). As Raman spectroscopy is a powerful probe to estimate strain, crystallinity, and chemical nature of the materials, it is utilized to characterise the initial growth of Ge on ceria as well as to study confinement of phonons in the growth direction of the film.

\section{Experimental details}

Raman intensity from the ultra thin films can be enhanced by employing an optical interference technique called IERS. ${ }^{10-14}$ This is basically an anti-reflection structure consisting of the following three layers: a bottom reflecting layer of aluminium, a middle transparent dielectric layer of ceria $\left(\mathrm{CeO}_{2}\right)$, and the top ultra thin layer of Ge which is to be investigated. The thickness of the ceria layer depends on the thickness of the Ge layer such that the reflection is minimum at the exciting laser wavelength. The calculations of the layer thicknesses have been done using the matrix method, ${ }^{15}$ wherein optical constants of $\mathrm{Ge}$ and $\mathrm{Al}$ were taken to be the same as that of their bulk form and refractive index of $\mathrm{CeO}_{2}$ layer is $2 \cdot 3$.

Aluminium films were prepared on Si substrates by thermal evaporation in a separate set-up. A hot cathode ion source of $3 \mathrm{~cm}$ diameter (Kaufman type) is used for sputter deposition of the films. $\mathrm{CeO}_{2}$ film is prepared by the reactive ion beam sputtering with $\mathrm{O}_{2}$ as the reactive gas. The Ar ions of energy $1 \mathrm{KeV}$ with a beam current of $14 \mathrm{~mA}(7 \mathrm{~mA})$ is employed to sputter the $\mathrm{CeO}_{2}(\mathrm{Ge})$ target. The partial pressures of $\mathrm{O}_{2}$ and $\mathrm{Ar}$ are $2.0 \times$ $10^{-4}$ mbar and $1.5 \times 10^{-4}$ mbar, respectively. Ceria films were prepared at a substrate temperature of $40^{\circ} \mathrm{C}$ and $\mathrm{Ge}$ films were prepared at substrate temperatures $\left(T_{s}\right)$ of $40^{\circ} \mathrm{C}$ and $300^{\circ} \mathrm{C}$. Raman spectra were recorded in the near backscattering geometry using DILOR XY spectrometer equipped with a CCD detector and 514.5 nm line of an argon ion laser as an excitation source (power of $\sim 50 \mathrm{~mW}$ ). AFM studies were done using the Digital Nanoscope-II in the contact mode. The nominal thicknesses of the films were initially estimated by the rate of deposition which is $4 \mathrm{~nm} / \mathrm{min}$ for ceria and $3 \mathrm{~nm} / \mathrm{min}$ for $\mathrm{Ge}$ film. Ultra thin layers of $\mathrm{Ge}$ of nominal thicknesses (1 to $10 \mathrm{~nm}$ ) were deposited on $\mathrm{CeO}_{2}$. The samples are named from $\mathrm{A}$ to $\mathrm{E}$ in the increasing order of nominal thickness: A (1 nm), B (2 nm), C (4 nm), D (7 nm) and E (10 nm).

\section{Results and discussion}

\subsection{Growth and morphology of Ge films}

Figure 1 shows the AFM measured surface features of germanium films A to E covering an area of $500 \times 500 \mathrm{~nm}$. The heights of the surface features are represented in gray 
(a)
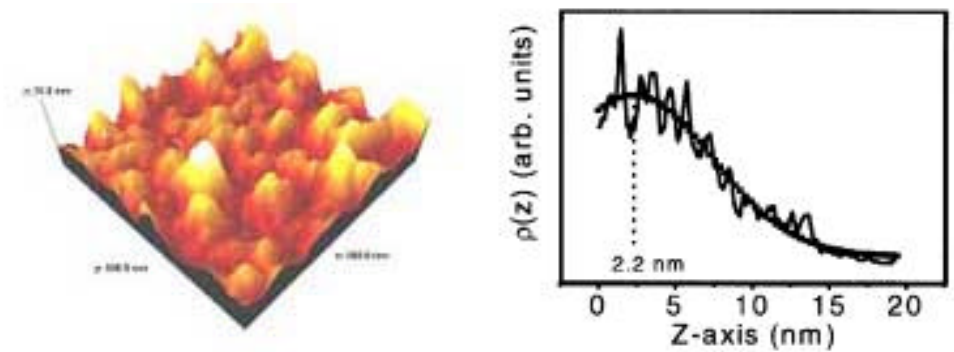

(b)
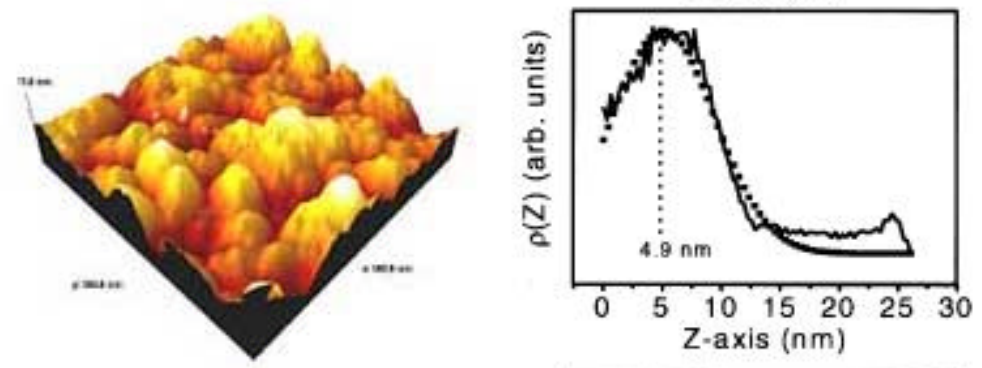

(c)
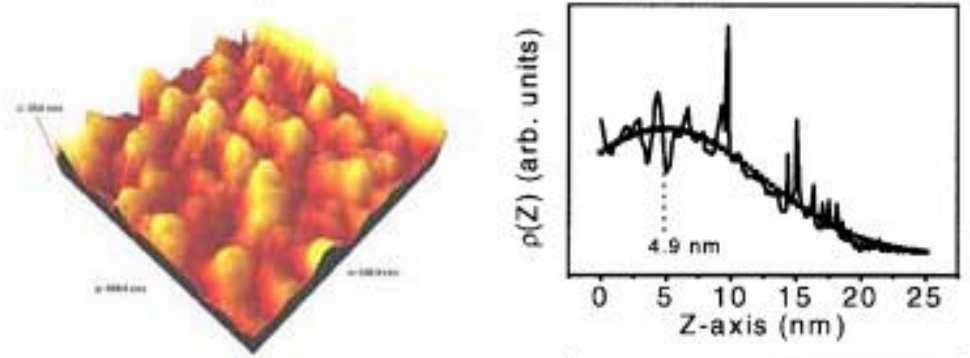

(d)
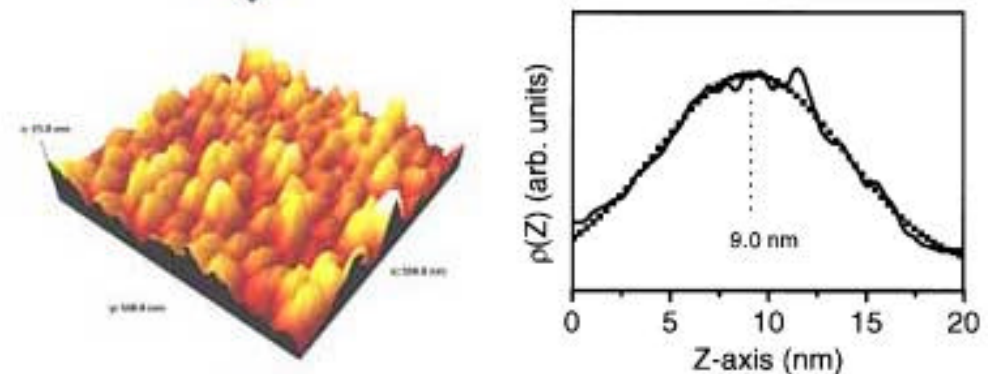

(e)
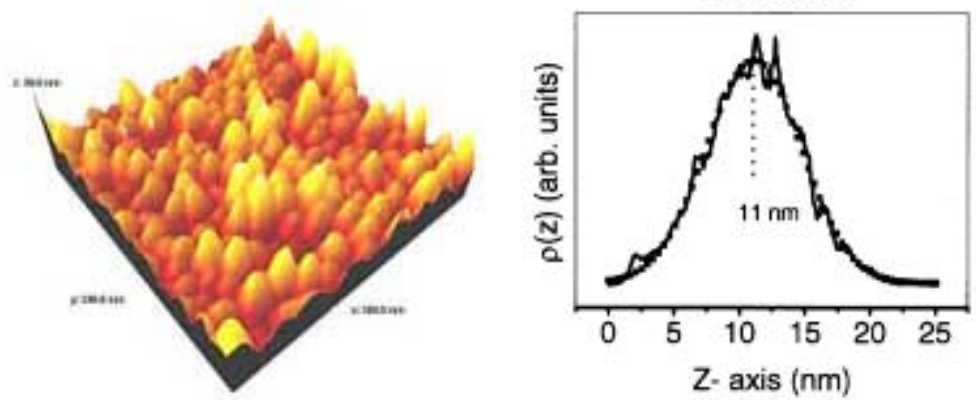

Figure 1. Surface morphology of the ultra thin Ge film samples A to E (left panels) and their corresponding height histograms (right panels). The dotted lines show the fitted Gaussian function. 
scale of 256 gradation with the brighter pixel indicating the top of hillock and the dark pixel indicating the valley. Figure 1 also shows the histogram of the heights $\rho(Z)$ in nanometers. Figure 2 indicates the line profile $S(x)$ and the corresponding auto-covariance

(a)

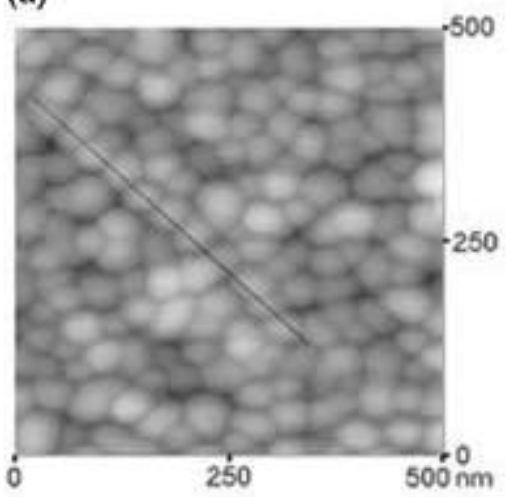

(b)

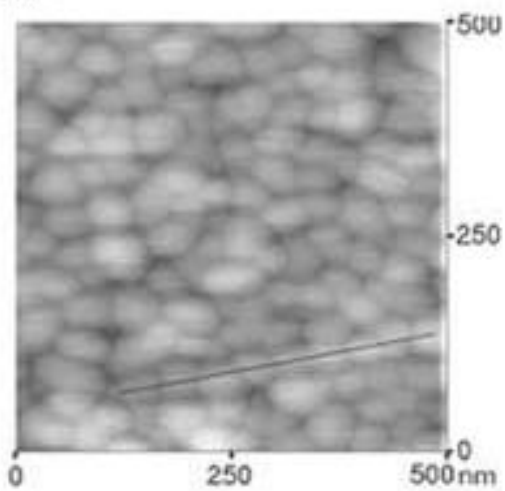

(c)

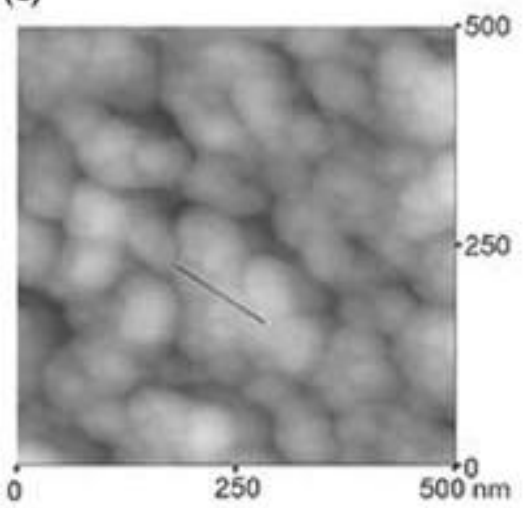

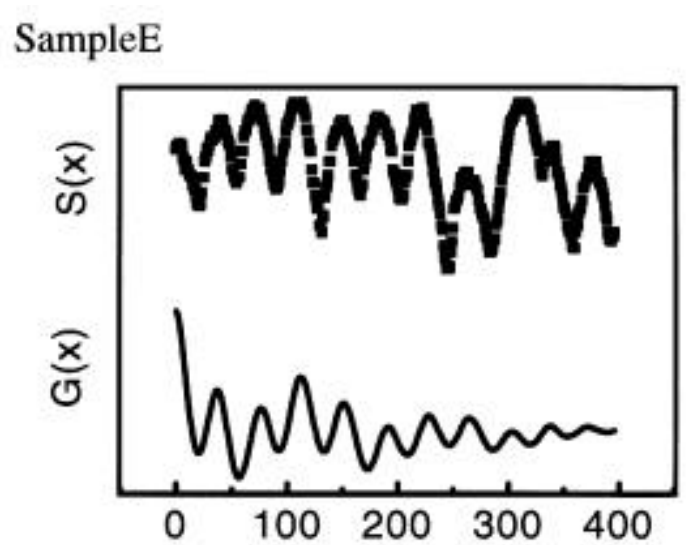

SampleD

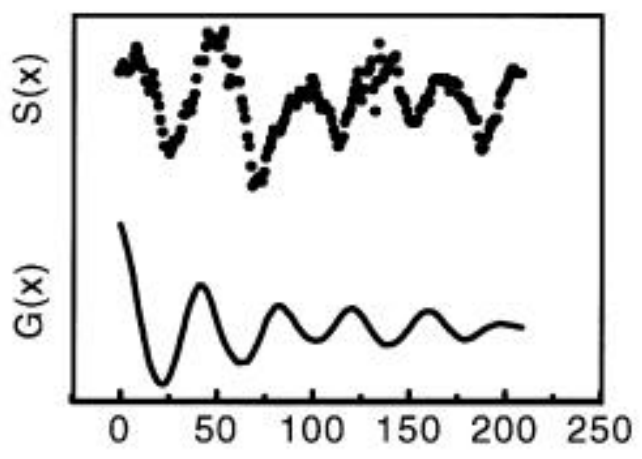

SampleB

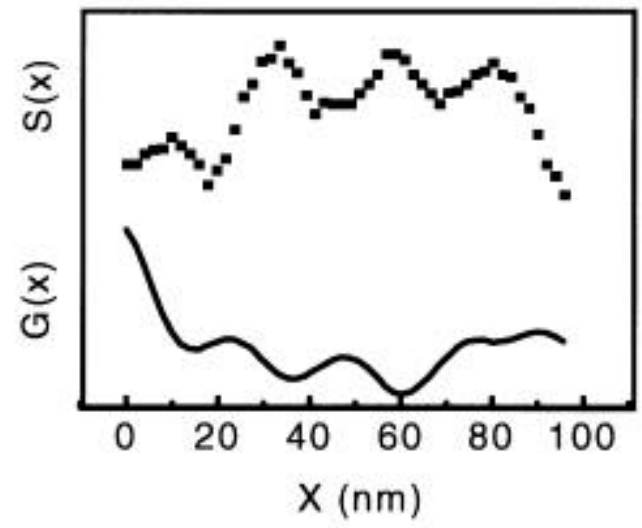

Figure 2. Left panels show two dimensional projections for the samples B, D and E. Right panels show the corresponding line profiles $S(x)$ and auto-covariance $G(x)$. 
$G(x)$ for the samples B, D and E along the line marked in the AFM scans (left panel). The AFM image of the sample A in figure 1 shows a large number of voids along with some clusters of Ge crystallites, which is an indication of a discontinuous Ge film. The regions corresponding to the voids are discarded and the regions where the Ge crystallites are present are shown in the histogram plot of figure 1 for each sample. The first moment of the Gaussian distribution fitted to the measured histogram gives the average thickness of the Ge crystallites to be $2.2 \mathrm{~nm}$ for the sample A. The grain sizes obtained from the AFM images were found to be $\sim 15-20 \mathrm{~nm}$. Sample B also shows a presence of voids. Figure 1 shows the average height of the crystallites as $4.9 \mathrm{~nm}$ and the average grain size is $\sim 20 \mathrm{~nm}$ (figure 2a). The small crystallites of $\mathrm{Ge}$ are observed across one single cluster. Sample C has more coverage of the Ge film with average thickness $4.9 \mathrm{~nm}$. Surface features of sample D and E confirm the formation of continuous Ge film with average thicknesses of 9 and $11 \mathrm{~nm}$, respectively and the average grain size is $\sim 40 \mathrm{~nm}$ (figure $2 \mathrm{~b}$ and c). From AFM studies, it is clear that during the initial growth of the film, cluster of 3D islands are formed. This is understandable because $\left(\sigma_{f}+\gamma_{i}\right)>\sigma_{s}$. The surface free energy $^{16}$ of $\mathrm{Ge}$ is $1.3 \mathrm{~J} / \mathrm{m}^{2}$ and $1.1 \mathrm{~J} / \mathrm{m}^{2}$ for ceria. ${ }^{17}$ Further, in the ion beam sputter deposition process, the sputtered Ge atoms come and deposit with energies $(\sim 10 \mathrm{eV})^{18}$ far greater than that of evaporation $(\sim 0.1 \mathrm{eV})$. This also contributes significantly to the growth of the films. These conditions favour the formation of 3D islands of Ge. This kind of growth mode, called Volmer-Webber growth mode, is also observed in the Ge films prepared on polymer substrates. ${ }^{2}$ It can be seen from figure 2 that the grain size of the film increases as deposition time is increased. The increase in the grain size can be attributed to the Ostwald ripening of the grains. ${ }^{1}$ The misfit strain gets relieved as the grains grow at the expense of the nearby smaller grains.

Figure 3 shows the structural changes (from amorphous to crystalline) in the Ge films of nominal thickness of $2 \mathrm{~nm}$. Ge film grown at $T_{s}$ of $40^{\circ} \mathrm{C}$ is amorphous as seen by a broad Raman mode centred at $270 \mathrm{~cm}^{-1}$ (figure $3 \mathrm{a}$ right panel). ${ }^{14}$ The amorphous film has a cluster size of $60 \mathrm{~nm}$ as shown in figure $3 \mathrm{a}$ (centre panel). Ge film prepared at $300^{\circ} \mathrm{C}$ is crystalline as revealed by the sharp Raman mode at $301 \mathrm{~cm}^{-1}$ (figure $3 \mathrm{~b}$ right panel). This film shows bigger clusters which are composed of smaller crystallites of size $\sim 20 \mathrm{~nm}$. This is inferred from the line profile $S(x)$ shown in figure $3 \mathrm{~b}$ (centre panel).

\subsection{Analysis of the Raman spectra}

Figure 4 shows interference enhanced Raman spectra of the samples A to E. Raman spectrum of bulk Ge single crystal is also shown for comparison purposes. Raman signal for the sample A was weak and therefore, nine point averaging was done to smoothen the spectrum. Raman spectra of all the samples (except sample B) show a red shift in the peak position and an asymmetrical broadening on the lower frequency side when compared with the spectrum of the bulk Ge sample. The shift of the Raman band in thin films with respect to its value in bulk Ge can arise due to tensile or compressive strain and phonon confinement. We will first estimate the shift due to phonon confinement (PC).

Raman line-shape for low-dimensional systems is given by, ${ }^{14,19-22}$

$$
I_{c}(\omega) \alpha \int_{0}^{q_{\max }} \frac{\mid c(q)^{2}}{[\omega-\omega(\vec{q})]^{2}+\Gamma_{0}^{2}} d^{3} q,
$$



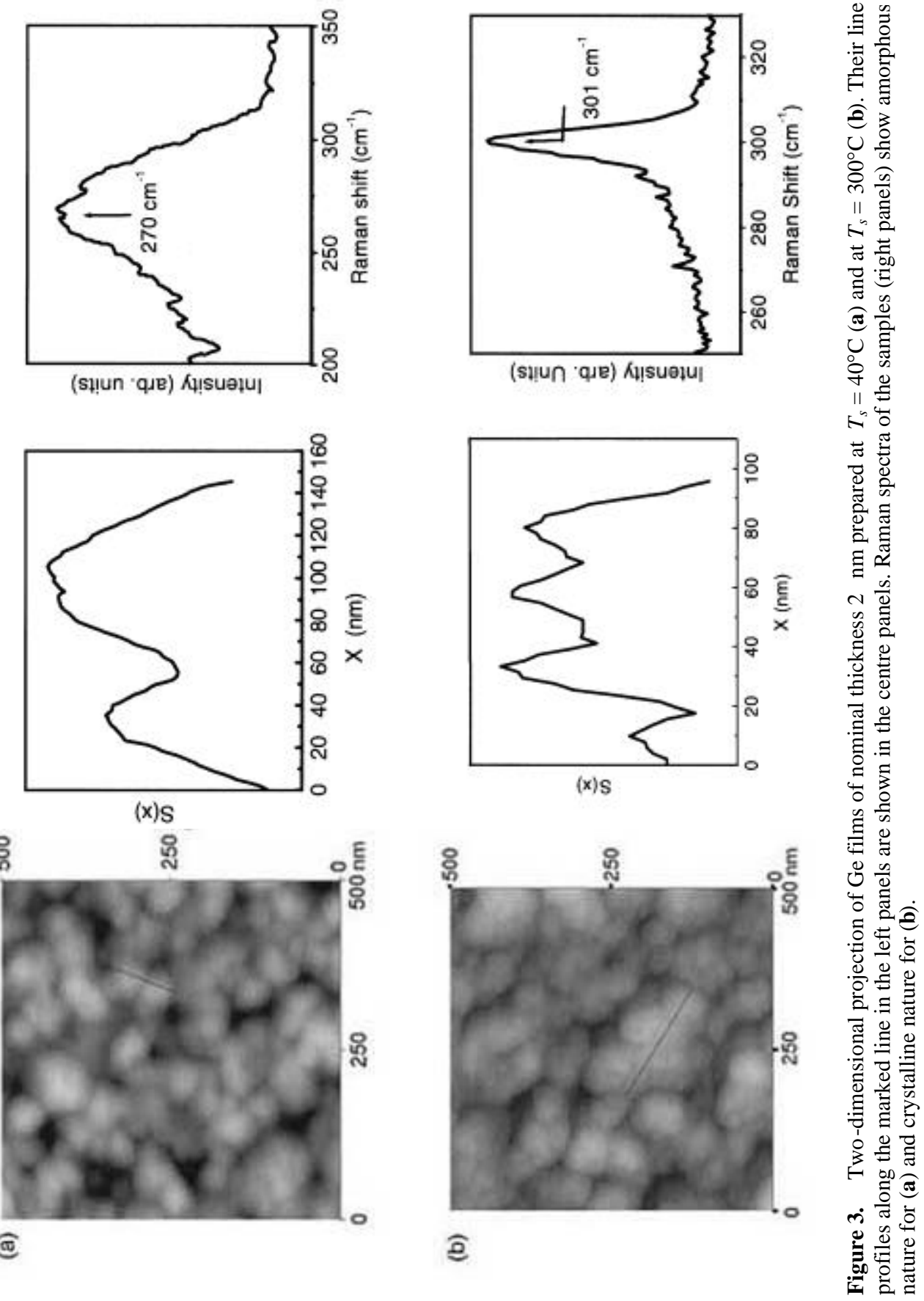


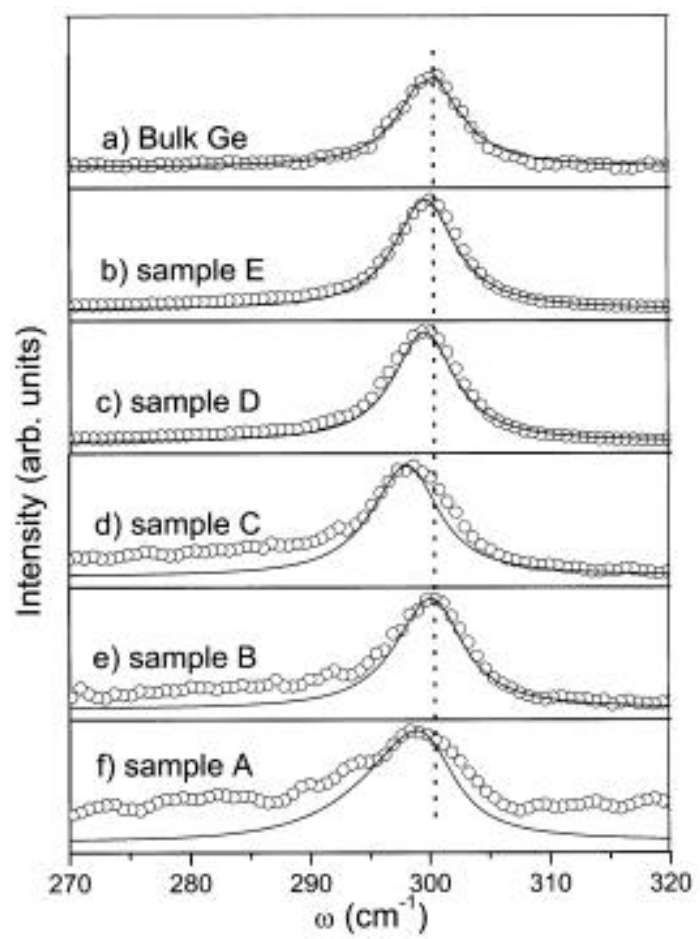

Figure 4. Shows observed Raman spectra (open circles) and the calculated spectra from the phonon confinement model (solid lines) for the samples A to E. Top panel shows the Raman spectrum of the bulk Ge. The calculated spectra for sample A and B are shifted up in the frequency to take into account the compressive strain, as explained in the text.

where $\omega(\vec{q})$ is the phonon dispersion curve of bulk Ge, $\Gamma_{\mathrm{o}}$ is the half width at half maximum (HWHM) of the Raman line of bulk Ge $\left(3.0 \mathrm{~cm}^{-1}\right)$ and $c(q)$ is the Fourier transform of the phonon weighting function W(L). In many cases, the Gaussian form of the phonon weighting function $\mathrm{W}(\mathrm{L})$ has been used and the corresponding $c(q)$ is given as $^{14,22}$

$$
|C(q)|^{2}=\frac{L}{\sqrt{32}} \cdot \frac{1}{8 \pi^{3}} \cdot \exp \left(\frac{-q^{2} L^{2}}{16 \pi^{2}}\right) \cdot\left|1-\operatorname{erf}\left(\frac{i q L}{\sqrt{32 \pi}}\right)\right|^{2}
$$

The phonon dispersion $\omega(\vec{q})$ along (111) direction is taken from the inelastic neutron scattering measurement ${ }^{23}$ and is same as used by us in ref. 12 and in ref. 14 (see inset in figure 5). Raman line shapes were calculated using (1) and (2) and the shift of the peak position $\Delta \omega\left(\Delta \omega=\omega_{\text {film }}-\omega_{\text {bulk }}\right)$ and full width at half maximum $2 \Gamma$ are extracted from the calculated spectra. Figure 5 (dashed lines) shows the calculated $\Delta \omega$ and $2 \Gamma$ as a function of film thickness L. Also shown in figure 5 (star symbols) are the experimental values for the five samples wherein the value of $\mathrm{L}$ is used as measured by AFM. It can be seen that the observed line-width agree well with the calculated values for all the 
samples, whereas the peak shifts agree only for samples C, D and E. The observed Raman peak positions for samples A and B are higher than the calculated values based on the phonon confinement model. We suggest that this due to the compressive strains because of the lattice mismatch between $\mathrm{Ge}$ and $\mathrm{CeO}_{2}$. The lattice constant of $\mathrm{CeO}_{2}$ $(5.41 \AA)$ is smaller by $\sim 4 \%$ than that of Ge $(5.65 \AA)$, which would result in a blue shift of $\sim 16 \mathrm{~cm}^{-1}$ of the Ge Raman line. ${ }^{24}$ However, the discrepancy between the observed peak position and the PC model is much smaller than this value. This is because the misfit strain can partially relax in a discontinuous or polycrystalline $\mathrm{Ge}$ film grown on a polycrystalline $\mathrm{CeO}_{2}$. For the layers of nominal thickness from 4 to $10 \mathrm{~nm}$ (sample $\mathrm{C}$ to $\mathrm{E})$, the growth changes to $3 \mathrm{D}$ islands and in the process relaxes the strain, thereby Raman shift for the samples C, D and E agreeing with the PC model.

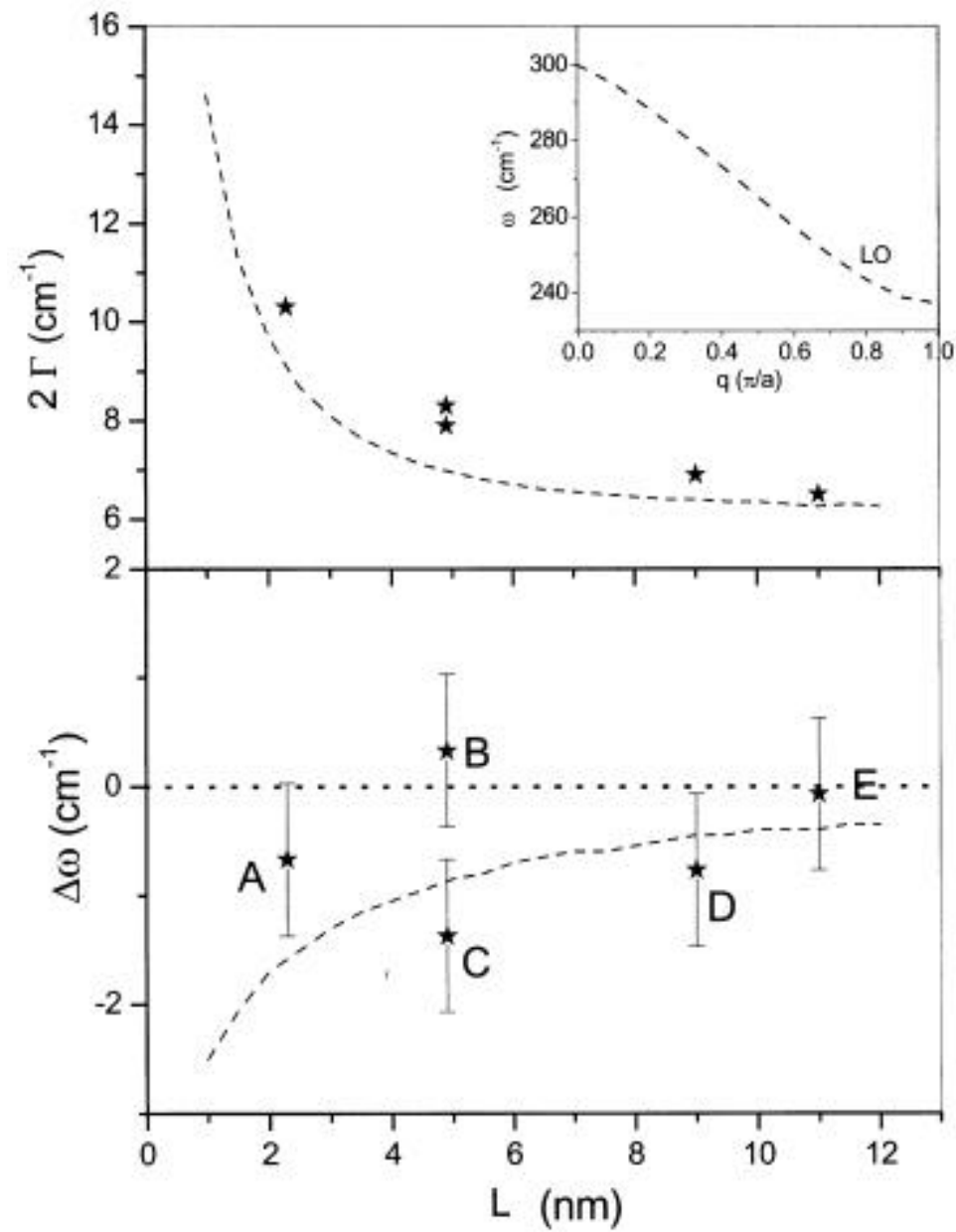

Figure 5. Shows the peak shift $\Delta \omega$ and line-width $2 \Gamma$ as a function of film thickness. Star symbol shows the observed peak shift, the line-width and the calculated values using (1) and (2) are shown by the dotted lines (Inset (ref. 23) shows the phonon dispersion curve used in the calculation). 


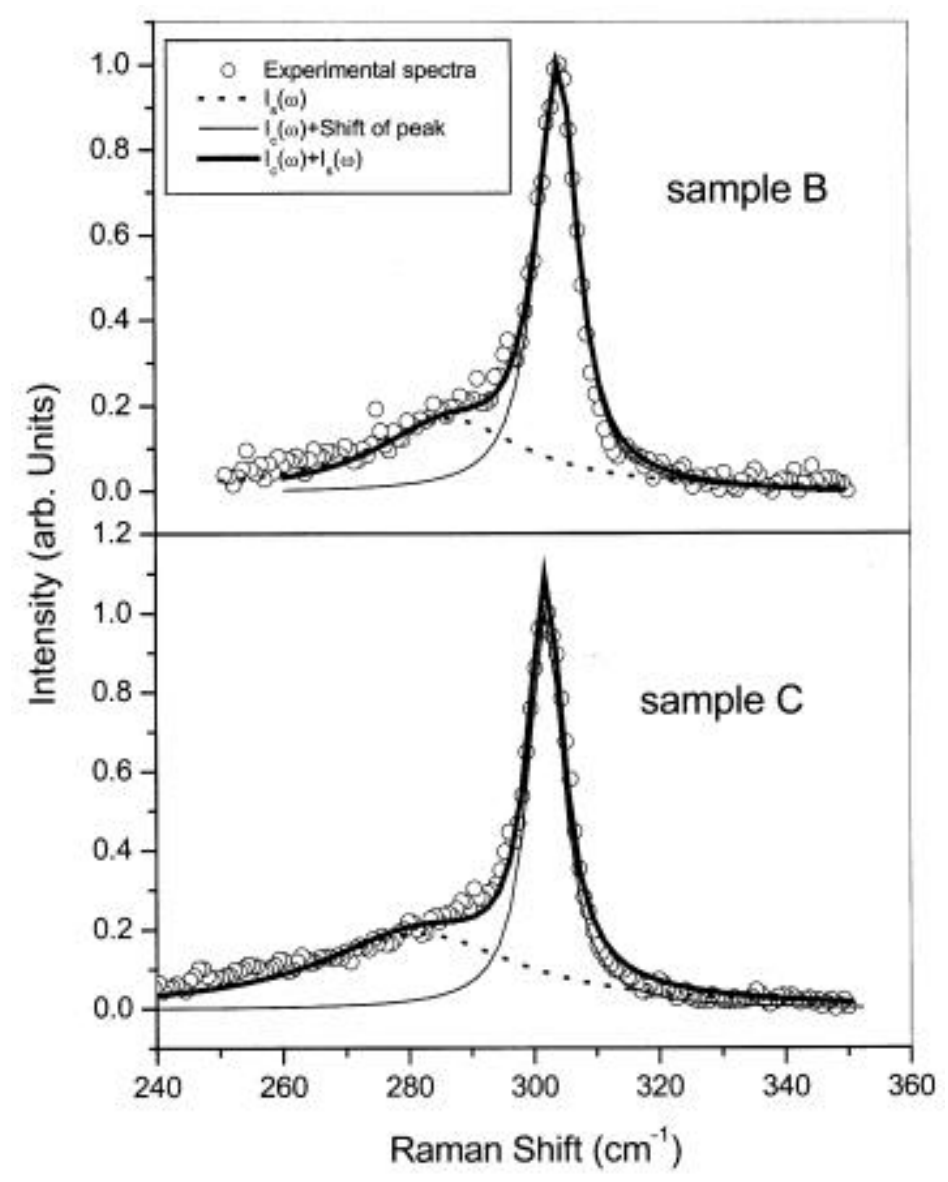

Figure 6. Raman spectra of samples B and C (open circles). The thick line is a resultant fit to $I_{c}(\omega)(1)$ (thin line) and a Lorentzian function (dotted line).

Figure 6 shows Raman spectra of the samples B and C. A shoulder at $280 \mathrm{~cm}^{-1}$ can be clearly observed on the lower frequency side of the main band. The observed spectra can be fitted (thick solid line) to a sum of $I_{c}(\omega)(1)$ and a Lorentzian centered at $280 \mathrm{~cm}^{-1}$ (dotted line). The component $I_{c}(\omega)$ has been shifted by $+1.2 \mathrm{~cm}^{-1}$ for sample B to take into account the compressive strain, as discussed above. The mode at $280 \mathrm{~cm}^{-1}$ is attributed to the surface phonon mode as discussed by Kanakaraju et al. ${ }^{14}$ The essential source for the surface mode contribution arises from the surface to volume ratio of the Ge nano-crystallites. The grain sizes as well as the coverage increases for the samples D and $\mathrm{E}$ and hence the surface phonon is not prominent in their Raman spectra. We have not fitted the Raman spectra of sample A due to poor signal to noise ratio.

\section{Conclusions}

Ultra thin crystalline Ge films of nominal thicknesses 1 to $10 \mathrm{~nm}$ were deposited by ion beam sputtering technique. The growth and morphology of the Ge films were studied by 
atomic force microscopy, which showed that the Ge films follow the Volmer-Weber growth mechanism. Ostwald ripening of the grains occur with an increase in the time of deposition. Raman spectra reveal that there is a compressive strain in samples A and B due to lattice mismatch between the Ge and ceria layers. Raman spectra also show a contribution from the surface phonon mode in samples B and C.

\section{Acknowledgements}

AKS thanks the Guest Editors for inviting him to contribute a paper to the special Festschrift Issue in honour of Prof. C N R Rao. We dedicate this paper to Prof. Rao on his 70th birthday and wish him many more years of exciting science and a healthy life. AKS and SM thank the Department of Science and Technology, Government of India, for financial assistance. We thank Dr Ganesan of Inter-University Consortium for DAE Facilities, Indore, for AFM measurements.

\section{References}

1. Gilberto Medeiros-Ribeiro, Bratkovski A M, Kamins T I, Ohlberg D A A and Williams S R 1998 Science 279 353; Bean J C 1985 Science 230127

2. Das A K, Kamila J, Dev B N, Sundaravel B and Kuri G 2000 Appl. Phys. Lett. 77951

3. Mo Y W, Savage D E, Swartzentruber B S and Lagally M G 1990 Phys. Rev. Lett. 651020

4. Wedler G, Walz J, Hesjedal T, Chilla E and Koch R 1998 Phys. Rev. Lett. 802382

5. Tomitotori M, Watanadbe K, Kobayashi M K, Iwawaki F and Nishikawa O 1994 Surface Sci. 301214

6. Walz J, Greuer A, Wedler G, Hesjedal T, Chilla V and Koch R 1998 Appl. Phys. Lett. 732579

7. Hammar M, LeGoues F K, Tersoff J, Reuter M C and Tromp R M 1996 Surface Sci. 349129

8. Ohring M 1992 The material science of thin films (California: Academic Press)

9. Celler G K and Sorin C 2003 J. Appl. Phys. 934956

10. Connell G A N, Nemanich R J and Tsai C C 1980 Appl. Phys. Lett. 3631

11. Nemanich R J, Tsai C C and Connel G A N 1980 Phys. Rev. Lett. 44273

12. Kanakaraju S, Sood A K and Mohan S 1998 J. Appl. Phys. 845756

13. Kanakaraju S, Sood A K and Mohan S 2000 Phys. Rev. B61 8334

14. Kanakaraju S, Sood A K and Mohan S 2001 Curr. Sci. 801550

15. Heavens O S 1955 Optical properties of thin solid films (London: Butterworths Scientific Publications)

16. Stekolnikov A A, Furthmuller J and Bechstedt F 2002 Phys. Rev. B65 115318

17. Baudin M, Wojcik M and Hermansson K 2000 Surface Sci. 46851

18. Cuomo J J, Rosanagel S M and Kaufman H R (eds) 1989 Hand book of ion beam processing technology (New Jersey: Noyes Publication)

19. Tuinstra F and Koenig J L 1970 J. Chem. Phys. 531126

20. Nemanich R J and Solin S A 1979 Phys. Rev. B20 329

21. Richter H, Wang Z P and Ley L 1981 Solid State Commun. 39625

22. Fauchet P M and Campbell I H 1988 Crit. Rev. Solid State Mater. Sci. 14 S 79

23. Nilsson G and Nelin G 1971 Phys. Rev. B3 364

24. Sutter P, Schwarz C, Muller E, Zelezny V, Gonacalves-Conto S and Von Kanel H 1994 Appl. Phys. Lett. 652220 